\title{
2008: year of the rat for stem cell research
}

\author{
Duanqing $\mathrm{Pei}^{1}$ \\ ${ }^{I}$ Stem Cell and Cancer Biology Group, CAS Key Laboratory of Regenerative Biology, South China Institute for Stem Cell Biology \\ and Regenerative Medicine, Guangzhou Institutes of Biomedicine and Health, Chinese Academy of Sciences, Guangzhou 510663, \\ China
}

Cell Research (2009) 19:149-151. doi: 10.1038/cr.2009.10; published online 2 February 2009

2008 breakthrough of the year went to reprogramming as announced by magazine Science recently [1], highlighting a stem cell revolution in the scientific world underway commencing at 2006. For the field of stem cell and developmental biology, 2008 ended with a truly exciting achievement for the Rat, i.e., the generation of germline competent embryonic stem cells from rat blastocysts (Figure 1) [2,3] .

This is significant because rat becomes only the second species that boosts germline competent ES cells after the mouse. Although ES-like cells have been reported for other species, including human and monkey, the pluripotency of those ES-like cells continues to be the subject of debate, due to the lack of chimera evidence - the established gold standard for pluripotency. Ethically, it is not feasible to demonstrate the pluripotency of human embryonic stem cells through chimera assay. Other species, on the other hand, should be suitable for chimera generation after ethical considerations. The question then appears to be why it took so long to generate truly pluripotent ES cells from other species. In the case of rat, it becomes apparent that the same technique used for mouse ES cell derivation is not adequate enough.

\footnotetext{
Correspondence: Duanqing Pei

Tel: 86-20-3229-0706; Fax: 86-20-3229-0606

E-mail: pei_duanqing@gibh.ac.cn
}

A landmark paper published by Ying and Smith in the 2008 May 22 issue of Nature paved the way [4]. Previously, the derivation and maintenance of pluripotent stem cells have been achieved mostly through empirical means. For example, mouse ES cells require the feeder layer and a combination of cytokines and growth factors to be maintained at the pluripotent state. It is known that these cells would undergo spontaneous differentiation if not maintained properly, a technical challenge proven to be quite frustrating for many investigators over the years. Through years of research on the self-renewal of mouse ES cells, the Smith lab has identified key regulators of ES cell selfrenewal, both extrinsic and intrinsic. It was thought that extrinsic factors such as LIF and other cytokines keep ES cells at the pluripotent state by activating intrinsic factors such as STAT3 required for ES cell self-renewal. Nevertheless, it is well known that ES cells have the tendency to differentiate in culture, making differentiation the number one enemy for self-renewal or pluripotency. Through a series of experiments, Ying and colleagues demonstrated that the extrinsic signals are not required for deriving or maintaining ES cells, thus, overcoming a huge conceptual hurdle. Furthermore, it was shown that the tendency for ES cells to differentiate in culture is largely mediated by signaling from the mitogen-activated protein kinases. By blocking MAPKs, the ES cells do not differentiate readily, but can not thrive well. This problem was solved by blocking the glycogen synthase kinase 3 . In the end, employing small chemical inhibitors for the MAPK and GSK3 pathways, Ying and colleagues were able to derive and propagate pluripotent ES cells, even from the STAT3-/background. Based on this publication, it is clear that ES cell derivation and maintenance have entered a rational phase from an empirical one. More importantly, the combined inhibition of MAPK and GSK3 pathways should become a universal approach in deriving and maintaining pluripotent ES cells not only from mouse blastocysts, but also from other species.

It came as no surprise that the original authors for the Nature paper achieved the fete for rat in parallel $[2,3]$. The key to their success is the utilization of inhibitors discovered in their original Nature paper that can block moues ES cell differentiation, and the same inhibitors worked well for rat ES cell derivation and self-renewal [2,3]. Both reports went through the most stringent test for ES cell pluripotency, i.e., the generation of live chimera and germline transmission of ES cell genomes to offsprings (Figure 1, left portion) [2,3]. With these germline competent ES cells, rat will become the next favorite model system for biomedical research after the mighty mouse. Some may argue that rat ES cells have been generated before these two reports. In fact, in 2008 alone, two 


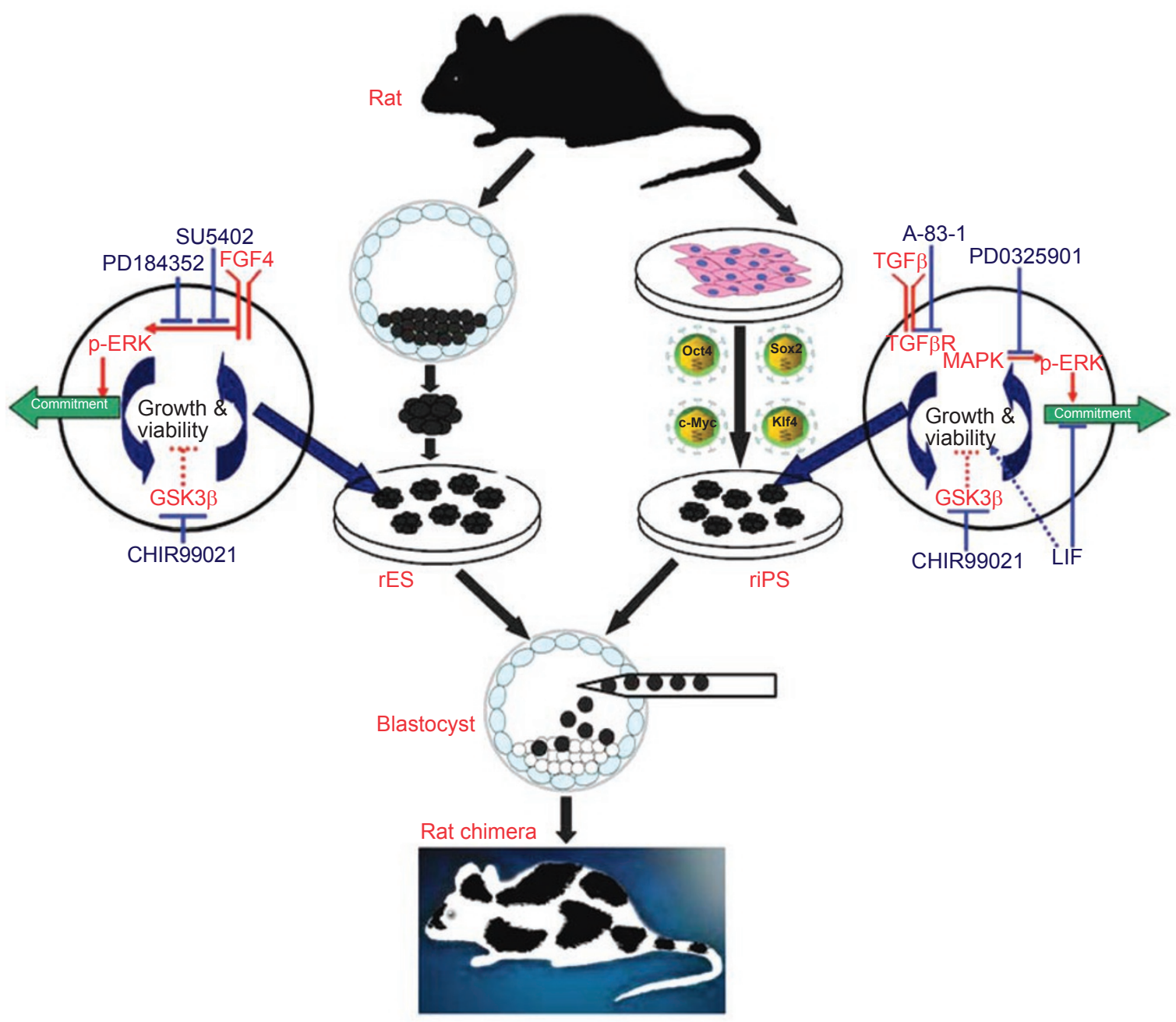

Figure 1 Generation of rES and riPS cell lines. On the left, the inner cell mass from blastocysts were cultured in the presence of chemical inhibitors to derive pluripotent embryonic stem cells ( $\mathrm{rES}$ ). On the right, rat adult cells were isolated and infected with a cocktail of viruses carrying reprogramming genes to genenrate iPS cells. Similar chemicals were added in culture media to ensure the generation of pluripotent cells.

other reports described the derivation of rat ES cells $[5,6]$ and these two reports were published ahead of those by Ying and Smith laboratories. It is significant that rat ES-like cells were derived by the Jin laboratory [5] and Ueda et al. [6] in a sense that both laboratories recognized the significance of rat as an experimental model and that no authentic ES cells had been generated before them. Although it is difficult to assess the technical differences between these methodologies, one assumes that the earlier reports did not fully address the differentiation problem intrinsic to the culture conditions without employing
MAPK and GSK3 inhibitors. It would be of great interest to see if the rES cells from the Jin laboratory and the Ueda group are also germline competent. If so, it would raise hopes that there are more than one way to generate and maintain rat $E S$ cells. If not, the rational approach employed by Ying and Smith laboratories may prove to be unique indeed.

The story does not stop at the ES lines. In two separate reports, rat induced pluripotent stem cells or rat iPS cells were described online (Figure 1, right portion) $[7,8]$. While Xiao and colleagues used the standard iPS approach for their rat iPS work [8], Ding and colleagues known for their clever use of small chemicals in maintaining ES cell culture, have succeeded in generating rat iPS cells with the help of a cocktail of chemicals [7]. Thus, a chemical approach to stem cell biology should have a bright future. Now, it would be of great interest to see if these rat iPS cells can go germline and prove to be as pluripotent as their ES counterparts or their mouse counterparts as well. Since reprogramming is considered the re-capture of lost pluripotency during differentiation [9], comparison between rat ES and iPS cells may reveal insights 
into reprogramming as shown by similar comparisons between mouse ES and iPS cells. To sum up, 2008 is certainly the year of the rat, but may also be remembered as the year of small chemicals for stem cell biology.

\section{Acknowledgment}

The author wishes to thank Mr Yi Gan for the illustration in Figure 1.

\section{References}

1 Alberts B. Celebrating a year of Science. Science 2008; 322:1757.
2 Buehr M, Meek S, Blair K, et al. Capture of authentic embryonic stem cells from rat blastocysts. Cell 2008; 135:12871298.

3 Li P, Tong C, Mehrian-Shai R, et al. Germline competent embryonic stem cells derived from rat blastocysts. Cell 2008; 135:1299-1310.

4 Ying QL, Wray J, Nichols J, et al. The ground state of embryonic stem cell selfrenewal. Nature 2008; 453:519-523.

5 Li C, Yang Y, Gu J, Ma Y, Jin Y. Derivation and transcriptional profiling analysis of pluripotent stem cell lines from rat blastocysts. Cell Res 2008 Nov 4. doi: 10.1038/cr.2008.301

6 Ueda S, Kawamata M, Teratani T, et al. Establishment of rat embryonic stem cells and making of chimera rats. PLoS ONE 2008; 3:e2800. doi:10.1371/journal.pone. 0002800

7 Li W, Wei W, Zhu S, et al. Generation of rat and human induced pluripotent stem cells by combining genetic reprogramming and chemical inhibitors. Cell Stem Cell 2008; 2008 Dec 17. doi:10.1016/j. stem.2008.11.014

8 Liao J, Cui C, Chen S, et al. Generation of induced pluripotent stem cell lines from adult rat cells. Cell Stem Cell 2008 Dec 17. doi:10.1016/j. stem.2008.11.013

9 Pei D. Regulation of pluripotency and reprogramming by transcription factors. J Biol Chem 2008 Sep 26. doi: 10.1074/ jbc.R800063200 